Check for updates

Cite this: RSC Adv., 2019, 9, 39294

Received 29th September 2019 Accepted 22nd November 2019

DOI: 10.1039/c9ra07900a

rsc.li/rsc-advances

\section{Circular RNA hsa_circ_0000467 modulates SGK1 to facilitate cell migration, metastasis, and EMT while repressing apoptosis in colorectal cancer by sponging $\mathrm{miR}-383-5 \mathrm{p}$}

\begin{abstract}
Chong Liu, ${ }^{a}$ Lingling Sun ${ }^{b}$ and Jiaying Sun (iD *b
Recent data indicated that circular RNAs (circRNAs) were implicated in tumor progression including colorectal cancer (CRC). However, the mechanism of hsa_circ_0000467 in CRC remains unclear. The levels of hsa_circ_0000467, microRNA-383-5p (miR-383-5p), and serum/glucocorticoid regulated kinase 1 (SGK1) in CRC tissues and cells were measured by quantitative real-time polymerase chain reaction ( $\mathrm{RRT}-\mathrm{PCR}$ ). The cell viability and apoptotic rate were detected through cell counting kit- 8 (CCK-8) assay and flow cytometry, respectively. The migration and invasion abilities were evaluated via Transwell assay. The protein levels of cleaved caspase 3 (C-caspase 3), B-cell lymphoma 2 (Bcl-2), Ncadherin, E-cadherin, SGK1, and proliferating cell nuclear antigen (PCNA) were detected by western blot assay. The dual-luciferase reporter assay and RNA immunoprecipitation (RIP) assay were constructed to verify the interaction between miR-383-5p and hsa_circ_0000467 or SGK1. The mouse model experiment was performed to further validate the effects of hsa_circ_0000467 on CRC progression. Hsa_circ_0000467 and SGK1 were enhanced while miR-383-5p was reduced in CRC tissues and cells. Hsa_circ_0000467 silencing suppressed cell proliferation, migration, invasion, and epithelialmesenchymal transition (EMT) but induced apoptosis in CRC cells by regulating miR-383-5p. Hsa_circ_0000467 sponged miR-383-5p and SGK1 was a direct target of miR-383-5p. Besides, hsa_circ_0000467 promoted SGK1 expression in CRC cells by sponging miR-383-5p. Furthermore, miR383-5p restrained cell proliferation, metastasis, and EMT but facilitated apoptosis in CRC cells by modulating SGK1. Also, hsa_circ_0000467 knockdown blocked xenograft tumor growth in vivo. Hsa_circ_0000467 promoted CRC progression by regulating SGK1 expression via miR-383-5p.
\end{abstract}

\section{Introduction}

Colorectal cancer (CRC) is a common malignant tumor and often accompanied with high morbidity and mortality. ${ }^{1}$ Despite the improvements in therapeutic approaches, such as surgical treatment, radiation, and chemotherapy, the 5 year survival rate of CRC patients is still unacceptable, due to the characteristics of local invasion and distant metastasis. ${ }^{2}$ Therefore, it is crucial to illustrate the mechanism of CRC progression.

Circular RNAs (circRNAs), a family of covalently closed loop structured RNA, have been reported to be related to tumor development and progression in diverse types of cancers, including CRC. ${ }^{3,4}$ For example, an exploration in lung cancer demonstrated that hsa_circ_0000064 was increased in lung

\footnotetext{
${ }^{a}$ Department of General Surgery, Shengiing Hospital Affiliated to China Medical University, China

${ }^{b}$ Pulmonary Department and Intensive Care Unit, The Fourth Affiliated Hospital of China Medical University, China Medical University, No. 4, Chongshan East Road, Huanggu District, Shenyang, Liaoning 110032, P. R. China. E-mail: ni5296237ue@ yeah.net; Tel: $+86-18900915346$
}

cancer, and its depletion constrained cell proliferation, but accelerated cell cycle attest and apoptosis. ${ }^{5}$ Jin et al. reported that hsa_circ_0136666 was enhanced in CRC, and its knockdown curbed cell proliferation, metastasis, and cell cycle progression in vitro and also retarded xenograft tumor growth in vivo. ${ }^{6}$ Also, the similar results of circ_0079993, ${ }^{7}$ circ_0104631, ${ }^{8}$ and circ_0005075 (ref. 9) were documented in CRC. Meanwhile, hsa_circ_0000467 was identified to involve in gastric cancer progression. ${ }^{10}$ However, the mechanism of hsa_circ_0000467 remains unknown in CRC.

MicroRNAs (miRNAs) are a form of small RNA ( $\sim 22$ nucleotides (nts)) with no translation capacity and affect gene expression through degrading message RNA (mRNA) or inhibiting mRNA translation. ${ }^{11}$ Many miRNAs were documented to associate with CRC progression, such as miR-1258, ${ }^{12}$ miR-837$5 \mathrm{p},{ }^{13}$ and $\mathrm{miR}-519 \mathrm{~b}-3 \mathrm{p}^{14}$ etc. Also, miR-383 was reported to be dysregulated in CRC and regulate CRC progression. ${ }^{15,16}$ Serum/ glucocorticoid regulated kinase 1 (SGK1), located on human chromosome 6q23.2, encodes a serine/threonine protein kinase which plays imperative roles in physiological processes in our 
body. ${ }^{17}$ Recent studies implied that the aberrant expression of SGK1 was also implicated in tumor progression and chemoresistance in various tumors, including CRC. ${ }^{18,19}$ Whereas, the mechanisms and functions of miR-383-5p and SGK were rarely documented in CRC. In the current research, we focused on the effects of hsa_circ_0000467 on CRC progression.

\section{Materials and methods}

\section{Tissues collection}

Thirty-five CRC tissue samples were obtained from Shengjing Hospital Affiliated to China Medical University, as well as pairing adjacent normal tissue samples. All tissues were frozen at $-80{ }^{\circ} \mathrm{C}$ until further used. Written informed consents were provided by all patients. This study was performed in strict accordance with the NIH guidelines for the care and use of laboratory animals (NIH Publication No. 85-23 Rev. 1985) and was approved by the Institutional Animal Care and Use Committee of Shengjing Hospital Affiliated to China Medical University.

\section{Cell culture and transfection}

Two CRC cell lines LoVo and HCT116 and normal colonic epithelial cell line NCM460 were purchased from Jining (Shanghai, China). All cells were cultured in McCoy's 5A medium (Solarbio, Beijing, China) supplemented with 10\% fetal bovine serum (FBS; Thermo Fisher Scientific, Rockville, $\mathrm{MD}$, USA) in an incubator with the parameters of $37^{\circ} \mathrm{C}$ and $5 \%$ $\mathrm{CO}_{2}$.

Small interfering RNA (siRNA) against hsa_circ_0000467 (sihsa_circ_0000467) and its negative control (si-NC), miR-383-5p mimics (miR-383-5p) and its control (NC), miR-383-5p inhibitor (anti-miR-383-5p) and its matched control (anti-NC) were obtained from GenePharma (Shanghai, China). Hsa_circ_0000467 and SGK1 were inserted into pLCDH-cir (Ribobio, Guangzhou, China) or pcDNA3.1 (Invitrogen, Carlsbad, CA, USA) plasmid to construct overexpression vector, namely pLCDHhsa_circ_0000467 or SGK1. The transfection was performed using Lipofectamine 2000 (Invitrogen).

\section{Quantitative real-time polymerase chain reaction (qRT-PCR)}

Total RNA was extracted using TriQuick Reagent (Solarbio), and the reverse transcription for circRNA/mRNA and miRNA was performed using miScript RT Kit (TaKaRa, Dalian, China) and a miRNA reverse transcription kit (Thermo Fisher Scientific), respectively. The qPCR was fulfilled using SYBR Premix Ex Taq II (TaKaRa), and glyceraldehyde 3-phosphate dehydrogenase (GAPDH) and small nuclear RNA U6 were chosen as internal references for circRNA/mRNA and miRNA, respectively. The levels of hsa_circ_0000467, miR-383-5p, and SGK1 in CRC tissues and cells were analyzed with the method of $2^{-\Delta \Delta C_{\mathrm{t}}}$. The oligonucleotides of primers were presented as follows: hsa_circ_0000467: (F, 5'-AATGGGACTTAAAAATGCGAGG-3' ${ }^{\prime}$, and R, 5'-GTTGTGGACTACGTGGAGACT-3'), miR-383-5p: (5'-GGGAGA TCAGAAGGTGATTGTGGCT-3' ${ }^{\prime}$, and R, 5'-CAGTGCGTGTCGTGGAGT-3'), SGK1: (F, 5'-AGGATGGGTCTGAACGACTTT-3' ${ }^{\prime}$, and R,
5'-TTTCCGATCACTTTCAAG-3'), GAPDH: (F, 5' -ACAGGGGAGGT GATAGCATT- $3^{\prime}$, and R, 5'-GACCAAAAGCCTTCATACATCTC- $3^{\prime}$ ), and U6: (F, 5'-ATTGGAACGATACAGAGAAGATT- ${ }^{\prime}$ ', and R, 5' GGAACGCTTCACGAATTTG-3').

\section{RNase $R$ resistance analysis of circRNA}

The total RNA samples were incubated with $3 \mathrm{U} \mathrm{mg}^{-1}$ RNase R (Geneseed, Guangzhou, China) for $30 \mathrm{~min}$ at $37^{\circ} \mathrm{C}$. Then, the treated RNA samples were reverse transcribed as above depicted. The expression of hsa_circ_0000467 and GAPDH in LoVo and HCT116 cells was tested by qRT-PCR.

\section{Cell counting kit-8 (CCK-8) assay}

The cell viability was measured by CCK-8 (Bioss, Beijing, China). The LoVo and HCT116 cells $\left(2 \times 10^{3}\right.$ per well $)$ were injected into a 96-well plate and cultivated for $24 \mathrm{~h}$. After transfection, LoVo and HCT116 cells were cultured for another $1 \mathrm{~d}, 2 \mathrm{~d}, 3 \mathrm{~d}$, or $4 \mathrm{~d}$, then CCK-8 solution was added into each well and incubated for $4 \mathrm{~h}$. The absorbance at $450 \mathrm{~nm}$ was detected through a microplate reader.

\section{Flow cytometry analysis of cell apoptosis}

Annexin V-Alexa Fluor 647/PI apoptosis detection kit (Fcmacs, Nanjing, China) was used to evaluate the apoptotic rate of LoVo and HCT116 cells. The cells $\left(1 \times 10^{5}\right)$ were re-suspended in binding buffer and then incubated with Annexin V-fluorescein isothiocyanate (FITC) for $10 \mathrm{~min}$, propidium iodide (PI) for another $5 \mathrm{~min}$ at room temperature in dark condition. The apoptotic rate of LoVo and HCT116 cells was evaluated through flow cytometry.

\section{Transwell assay}

The Transwell chamber (Corning, Tewksbury, MA, USA) was utilized to assess the migrated and invaded abilities of LoVo and HCT116 cells. For cell migration, the lower Transwell chamber was added with McCoy's 5A medium containing 10\% FBS, while the upper one was injected with LoVo or HCT116 cells in McCoy's 5A medium without FBS. Following $24 \mathrm{~h}$ cultivation, the migrated cells were fixed with $4 \%$ methanol and stained with $0.1 \%$ violet crystal. The cells in 5 randomly selected fields were counted under a microscope. For cell invasion, the difference is the upper chamber was pre-plated with Matrigel matrix (Solarbio).

\section{Western blot assay}

The protein in LoVo and HCT116 cells was extracted using RIPA reagent (Solarbio), and the concentration of protein samples was measured using a BCA detection kit (Beyotime, Shanghai, China). Then sodium dodecyl sulfonate-polyacrylamide gel electrophoresis (SDS-PAGE) was used to separate protein samples, and then the bands were transferred onto a polyvinylidene fluoride (PVDF) membrane (Millipore, Billerica, MA, USA). Subsequently, the membrane was sealed in skim milk for $4 \mathrm{~h}$ at $37{ }^{\circ} \mathrm{C}$, then incubated with primary antibody for $12 \mathrm{~h}$ at $4{ }^{\circ} \mathrm{C}$ and secondary antibody for $2 \mathrm{~h}$ at $37^{\circ} \mathrm{C}$. The bands' 
intensities were tested using an ECL kit (Beyotime). The cleavedcaspase 3 (C-caspase 3, 1:500), B-cell lymphoma 2 (Bcl-2, $1: 1000)$, N-cadherin $(1: 1000)$, E-cadherin $(1: 500)$, SGK1 $(1: 1000)$, PCNA $(1: 1000)$, and GAPDH $(1: 10$ 000) primary antibodies and goat anti-rabbit secondary antibody IgG ( 1 : 10 000) were obtained from Abcam (Cambridge, MA, USA).

\section{Dual-luciferase reporter assay}

The interaction between miR-383-5p and hsa_circ_0000467 or SGK1 was predicted by starbase v3.0 (http://starbase.sysu.edu.cn/ ). The wild type (containing complementary fragments) or mutant sequences of hsa_circ_0000467 or 3'-untranslated regions $\left(3^{\prime} \mathrm{UTR}\right)$ of SGK1 were inserted into pmirGLO vector (Youbio, Changsha, China) to construct luciferase reporter, namelyhsa_circ_0000467-wt, hsa_circ_0000467-mut, SGK1-wt, or SGK1-mut. The Lipofectamine 2000 was used for the cotransfection of luciferase reporter and miR-383-5p or NC. The luciferase activity was evaluated using Dual-Lucy Assay Kit (Solarbio).

\section{RNA immunoprecipitation (RIP) assay}

A RIP Kit (Millipore) was used to analyze the binding specificity between miR-383-5p and hsa_circ_0000467 or SGK1. Briefly, LoVo and HCT116 cells were firstly lysed in RIP lysis buffer containing protease and ribonuclease inhibitors.
Subsequently, $100 \mu \mathrm{L}$ cell lysate sample was cultured with RIP buffer containing magnetic beads labeled with Anti-Ago2 or Anti-IgG. Then the mixture was incubated with protease $\mathrm{K}$ to digest the protein content. The enrichments of hsa_circ_0000467, miR-383-5p, and SGK1 were examined by qRTPCR.

\section{Mice xenograft model}

The nude mice experiment was performed according to the procedures approved by the Animal Care Committee of Shengjing Hospital Affiliated to China Medical University. The HCT116 cells $\left(6 \times 10^{6}\right)$ stably transfected with sh-NC or shhsa_circ_0000467 were implanted into six-week-old male nude mice ( $n=6$ per group). Following injection, the volume of xenograft tumor was measured every 5 days for 5 times and calculated according to the formula: volume $\left(\mathrm{mm}^{3}\right)=$ width $^{2} \times$ length/2. At $25 \mathrm{~d}$ measurement, the xenograft tumors were resected from nude mice, and the xenograft tumors' weights were measured. The tumors were frozen for the next study.

\section{Statistical analysis}

GraphPad Prism 7 (GraphPad Inc., La Jolla, CA, USA) was used to analyze the experimental data. All quantitative data from three independent experiments were presented as the mean \pm standard deviation (SD). The differences between the two
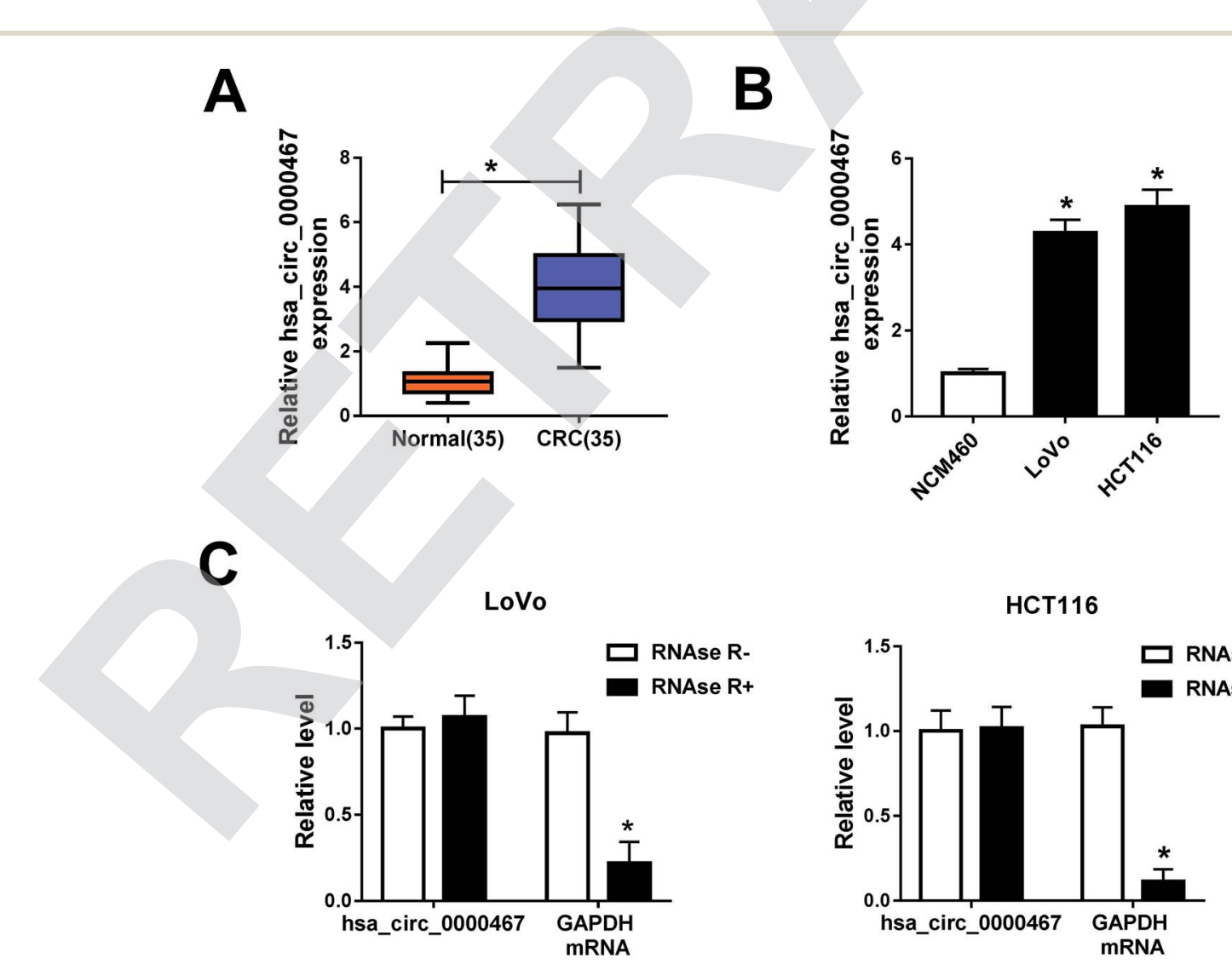

Fig. 1 Hsa_circ_0000467 was elevated in CRC tissues and cells. (A and B) The level of hsa_circ_0000467 in CRC tissues and cells was examined by qRT-PCR. (C) The levels of hsa_circ_0000467 and GAPDH in RNAse R (+/-)-treated LoVo and HCT116 cells were tested via qRT-PCR. *P < 0.05 . 
groups were processed by Student's $t$-test, while among multiple groups were estimated by one-way analysis of variance (ANOVA). $P<0.05$ was considered as statistical significance.

\section{Results}

\section{Hsa_circ_0000467 was elevated in CRC tissues and cells}

In order to investigate the effects of has_circ_0000467 on CRC progression, the level of hsa_circ_0000467 was firstly measured in CRC tissues. As shown in Fig. 1A, hsa_circ_0000467 was dramatically up-regulated in CRC tissues related to that in adjacent normal tissues. Also, the level of hsa_circ_0000467 was obviously increased in CRC cell lines LoVo and HCT116 compared with that in normal colonic epithelial cell line NCM460 (Fig. 1B). To verify hsa_circ_0000467 was a circRNA, RNAse R was used to treat the LoVo and HCT116 cells. As exhibited in Fig. 1C, the level of hsa_circ_0000467 had no striking change in any group, while the level of GAPDH was apparently declined in RNAse R-treated group. These data implied that hsa_circ_0000467 was a circRNA and remarkably augmented in CRC tissues and cells.
Hsa_circ_0000467 knockdown constrained cell proliferation, migration, invasion, and EMT while facilitated apoptosis in LoVo and HCT116 cells

To elucidate the functions of hsa_circ_0000467 in CRC progression, hsa_circ_0000467 knockdown was studied in CRC cells. The knockdown efficiency was confirmed, demonstrated by the marked reduction of hsa_circ_0000467 in LoVo and HCT116 cells transfected with si-hsa_circ_0000467 in contrast to that in negative control group (Fig. 2A). Moreover, the cell viability was distinctly decreased in si-hsa_circ_0000467transfected LoVo and HCT116 cells (Fig. 2B). However, the transfection of si-hsa_circ_0000467 contributed to the upregulation of apoptotic rate in LoVo and HCT116 cells (Fig. 2C). The Transwell assay indicated that the migrated and invaded abilities were both conspicuously decreased in LoVo and HCT116 cells transfected with si-hsa_circ_0000467 (Fig. 2D and E). Since C-caspase 3, Bcl-2 as pro-apoptosis-related factors, and $\mathrm{N}$-cadherin, E-cadherin as epithelial-mesenchymal transition (EMT)-associated protein markers, we measured the levels of these protein in LoVo and HCT116 cells. As exhibited in
A

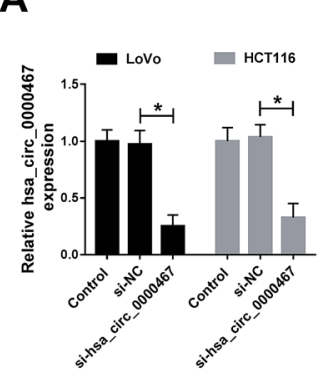

D

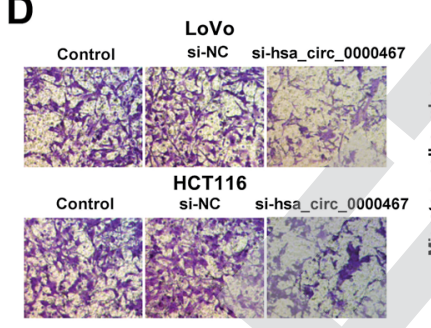

$\mathbf{F}$

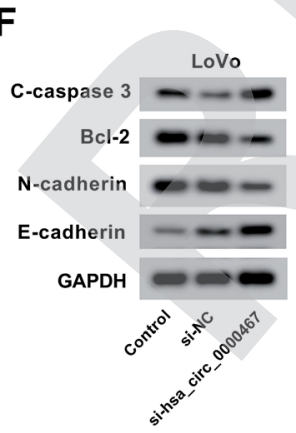

B

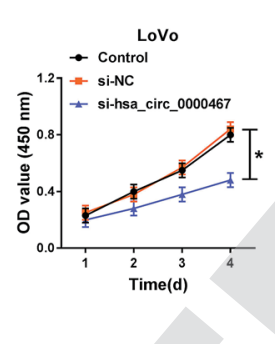

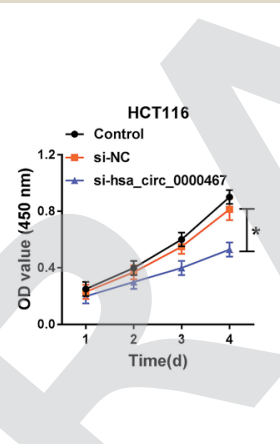

C

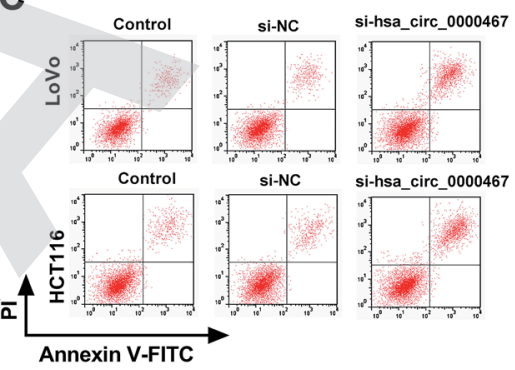

E
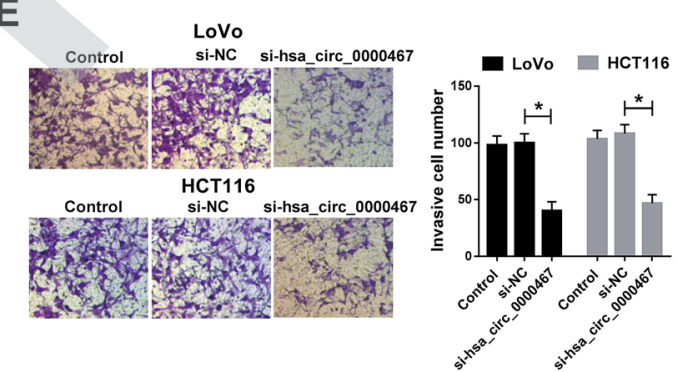
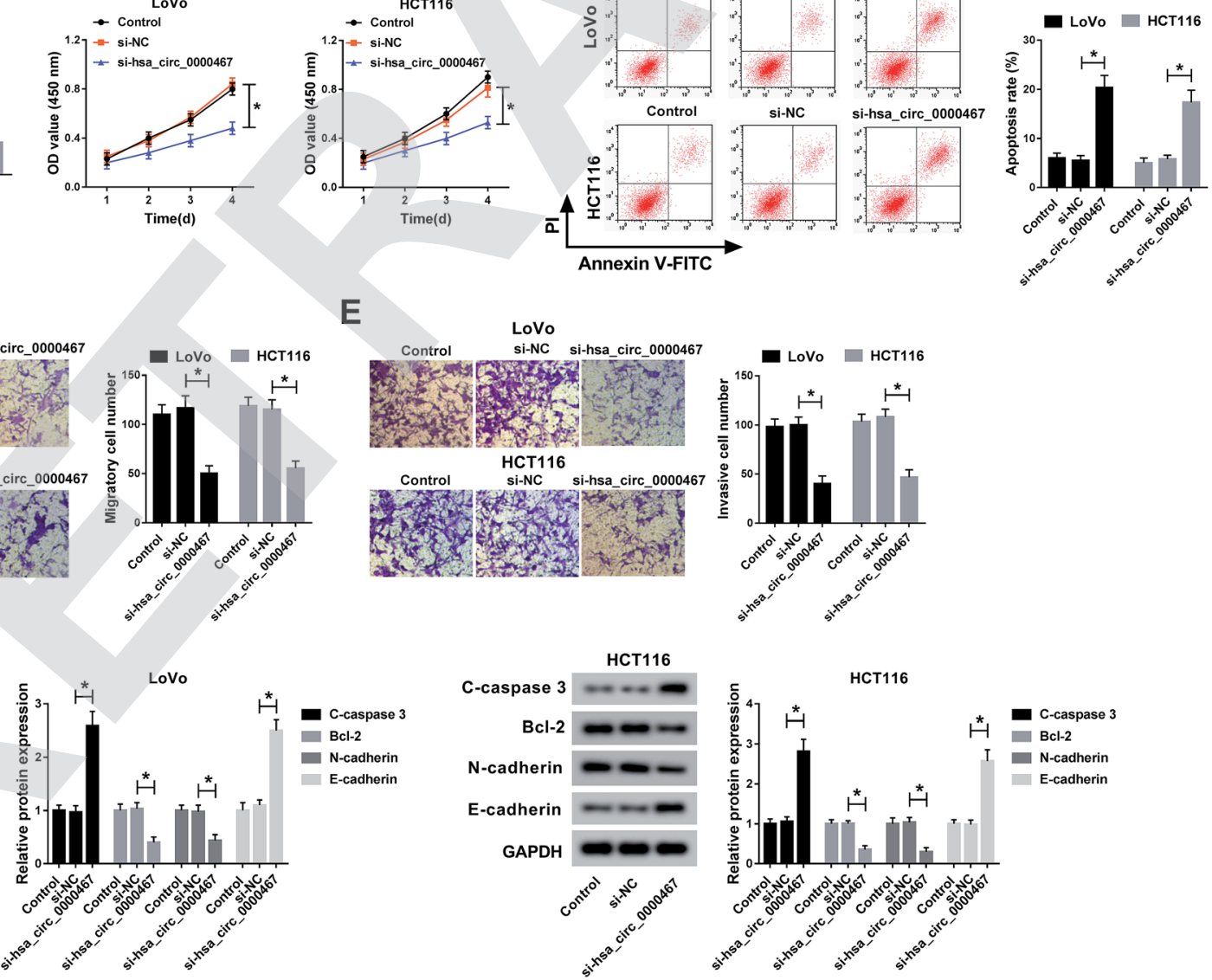

Fig. 2 Hsa_circ_0000467 knockdown constrained cell proliferation, migration, invasion, and EMT while facilitated apoptosis in LoVo and HCT116 cells. (A-F) The LoVo and HCT116 cells were transfected with si-hsa_circ_0000467 or negative controls. (A) The level of hsa_circ_0000467 was tested via qRT-PCR. (B) The cell viability was monitored by CCK- 8 assay. (C) The apoptotic rate was assessed through flow cytometry. ( $D$ and E) The migrated and invaded cell numbers were calculated via Tranwell assay. (F) The protein levels of C-caspase 3, Bcl-2, Ncadherin, and E-cadherin were detected by western blot assay. ${ }^{*} P<0.05$. 
Fig. 2F, the introduction of si-hsa_circ_0000467 apparently elevated the protein levels of C-caspase 3, E-cadherin, while greatly retarded the protein levels of Bcl-2, N-cadherin in LoVo and HCT116 cells. Taken together, hsa_circ_0000467 silencing confined cell proliferation, migration, invasion, and EMT while facilitated apoptosis in CRC cells.

\section{Hsa_circ_0000467 sponged miR-383-5p in LoVo and HCT116 cells}

Recent exploration indicated that miR-383-5p functioned as a tumor suppressor in diverse cancers. The scatter presented that hsa_circ_0000467 was negatively linear correlated with miR-3835p (Fig. 3A). Also, miR-383-5p was effectively down-regulated in CRC tissues and cells (Fig. 3B and C). Besides, the level of miR383-5p was notably decreased in LoVo and HCT116 cells transfected with pLCDH-hsa_circ_0000467, while drastically enhanced in si-hsa_circ_0000467-transfected LoVo and HCT116 cells (Fig. 3D). Through the starBase v3.0 online database, miR383-5p had complementary base pairing with hsa_circ_0000467 (Fig. 3E). Furthermore, the following dual-luciferase reporter assay exhibited that the transfection of miR-383-5p contributed to the distinct decline of luciferase activity of hsa_circ_0000467wt reporter compared to that in NC group, while the luciferase activity of hsa_circ_0000467-mut reporter had no significantly change in any group (Fig. 3F).In addition, Anti-Ago2 enriched much more hsa_circ_0000467 or miR-383-5p in LoVo and HCT116 cells in comparison with that in Anti-IgG group (Fig. 3G). To sun up, hsa_circ_0000467 was negatively interacted with miR383-5p in LoVo and HCT116 cells.

Hsa_circ_0000467 silencing refrained cell proliferation, metastasis, and EMT but induced apoptosis in LoVo and HCT116 cells by regulating miR-383-5p

To elucidate whether hsa_circ_0000467 regulating cell behaviors in CRC cells was mediated by miR-383-5p, sihsa_circ_0000467 and anti-miR-383-5p were transfected into LoVo and HCT116 cells. Firstly, the level of miR-383-5p was apparently augmented in si-hsa_circ_0000467-transfected LoVo and HCT116 cells, while partly reduced by the re-introduction of anti-miR-383-5p (Fig. 4A). Also, the transfection of sihsa_circ_0000467 resulted in the distinct decrease of cell viability, migrated and invaded abilities in LoVo and HCT116
A

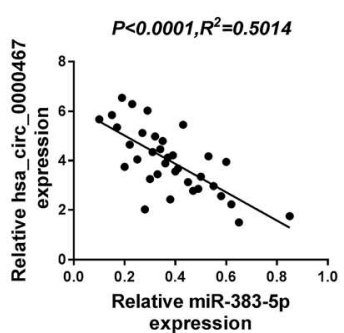

D

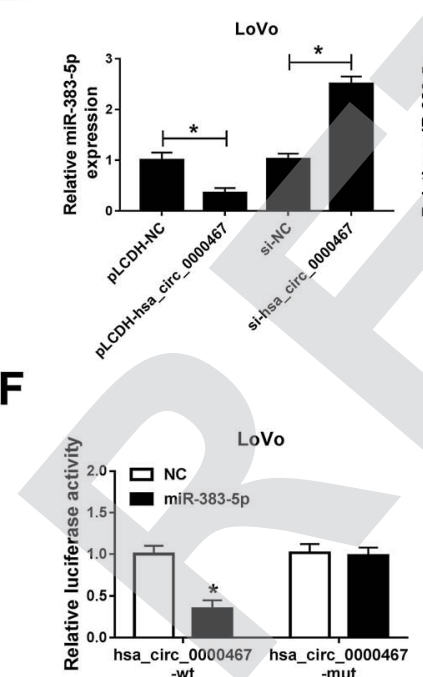

B

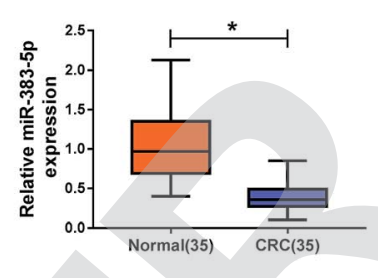

C

E
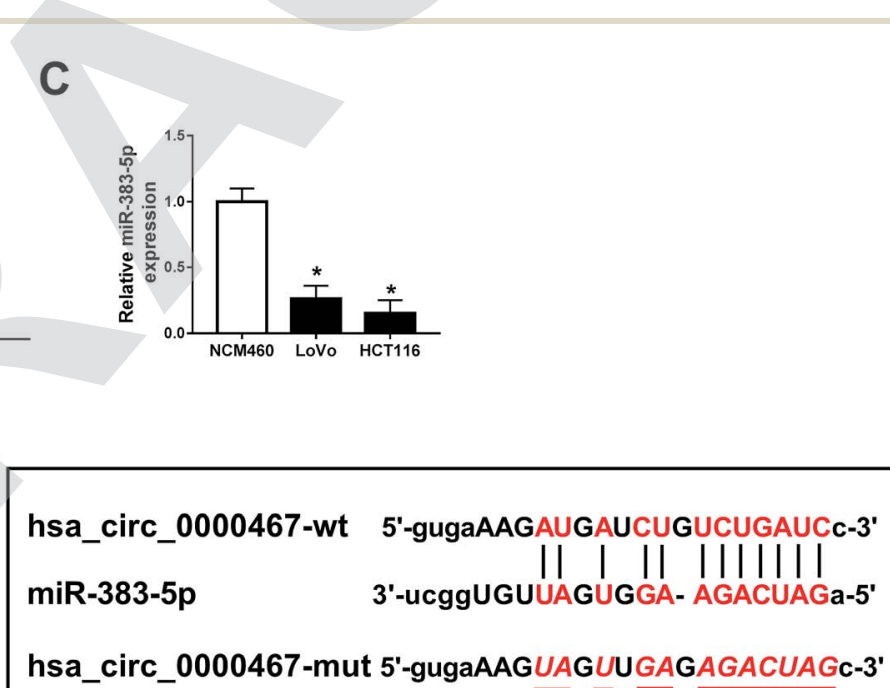

G
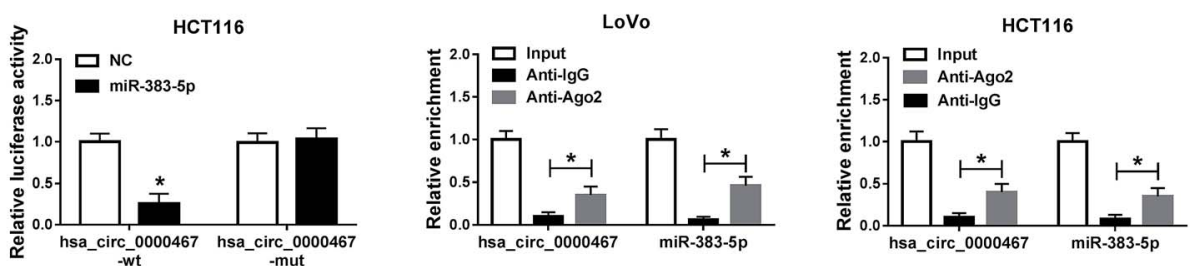

Fig. 3 Hsa_circ_0000467 sponged miR-383-5p in LoVo and HCT116 cells. (A) The correlation between levels of hsa_circ_0000467 and miR383-5p was analyzed by Pearson test. (B and C) The level of miR-383-5p in CRC tissues and cells was examined via qRT-PCR. (D) The level of miR-383-5p in LoVo and HCT116 cells transfected with pLCDH-NC, pLCDH-hsa_circ_0000467, si-NC, or si-hsa_circ_0000467 was tested by qRT-PCR. (E) The complementary sequences between hsa_circ_0000467 and miR-383-5p were exhibited, as well as the mutant sequences of hsa_circ_0000467. (F) The luciferase activity of hsa_circ_0000467-wt or hsa_circ_0000467-mut reporter in LoVo and HCT116 cells transfected with miR-383-5p or NC was evaluated via dual-luciferase reporter assay. (G) The enrichment of hsa_circ_0000467 or miR-383-5p was detected via RIP assay. $* P<0.05$. 
cells, while regained in the LoVo and HCT116 cells cotransfected si-hsa_circ_0000467 and anti-miR-383-5p (Fig. 4B, $\mathrm{D}$, and E). However, the apoptotic rate in LoVo and HCT116 cells showed the opposite trends. In brief, the apoptotic rate was strikingly enhanced in si-hsa_circ_0000467 group, while the emergence of anti-miR-383-5p mitigated this promoted effect (Fig. 4C). Besides, the introduction of anti-miR-383-5p counteracted the accelerated impact on the protein levels of $\mathrm{C}$ caspase 3, E-cadherin, as well as the restraint impact on the protein levels of Bcl-2, N-cadherin in LoVo and HCT116 cells caused by si-hsa_circ_0000467 (Fig. 4F and G). These data implied that hsa_circ_0000467 knockdown suppressed cell proliferation, migration, invasion, and EMT while facilitated apoptosis in CRC cells by modulating miR-383-5p.

\section{SGK1 was negatively interacted with miR-383-5p in LoVo and} HCT116 cells

Emerging evidence showed that SGK1 acted as an oncogene in various types of cancers. ${ }^{20}$ The scatter diagram exhibited SGK1 was negatively correlated with miR-383-5p, while positively correlated with hsa_circ_0000467 (Fig. 5A and B). Besides, the mRNA and protein levels of SGK1 were highly expressed in CRC tissues and cells (Fig. 5C and E). The protein level of SGK1 was significantly declined in miR-383-5p-transfected LoVo and HCT116 cells, but regained by the re-introduction of pLCDHhsa_circ_0000467 (Fig. 5F). As shown in Fig. 5G, starBase v3.0 predicted that the $3^{\prime}$ UTR of SGK1 had complementary binding sites with miR-383-5p. Following dual-luciferase reporter assay implied that the luciferase activity of SGK1-wt reporter was
A
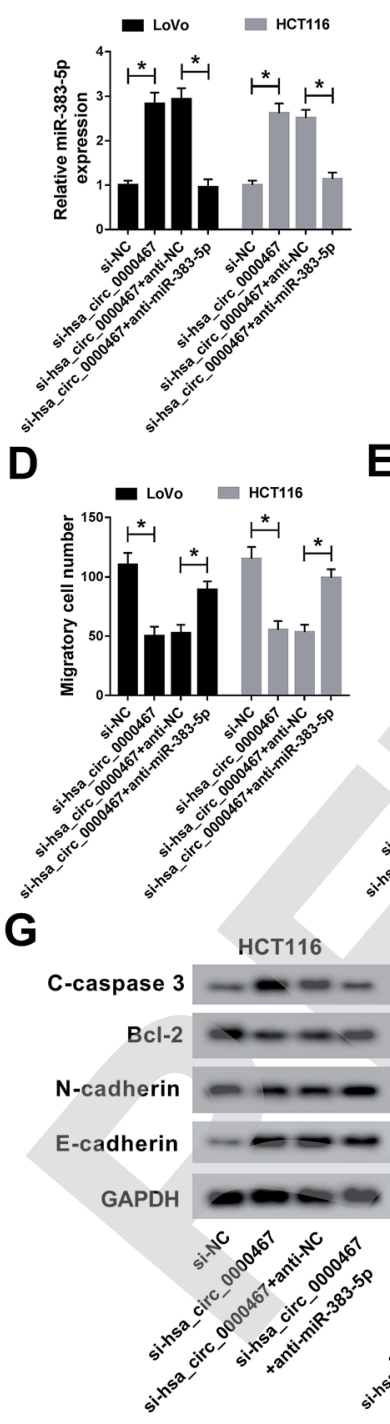

B

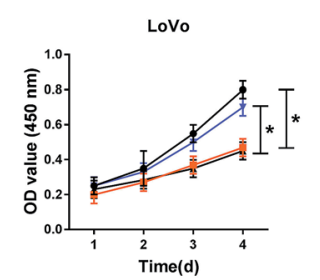

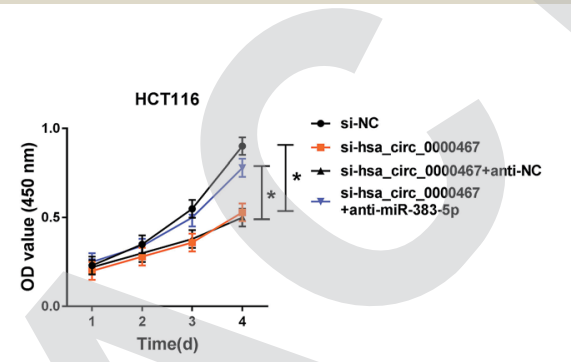

C

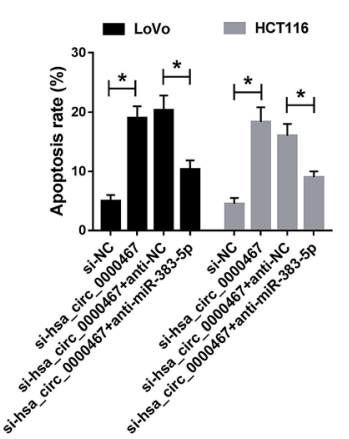

$\mathbf{F}$
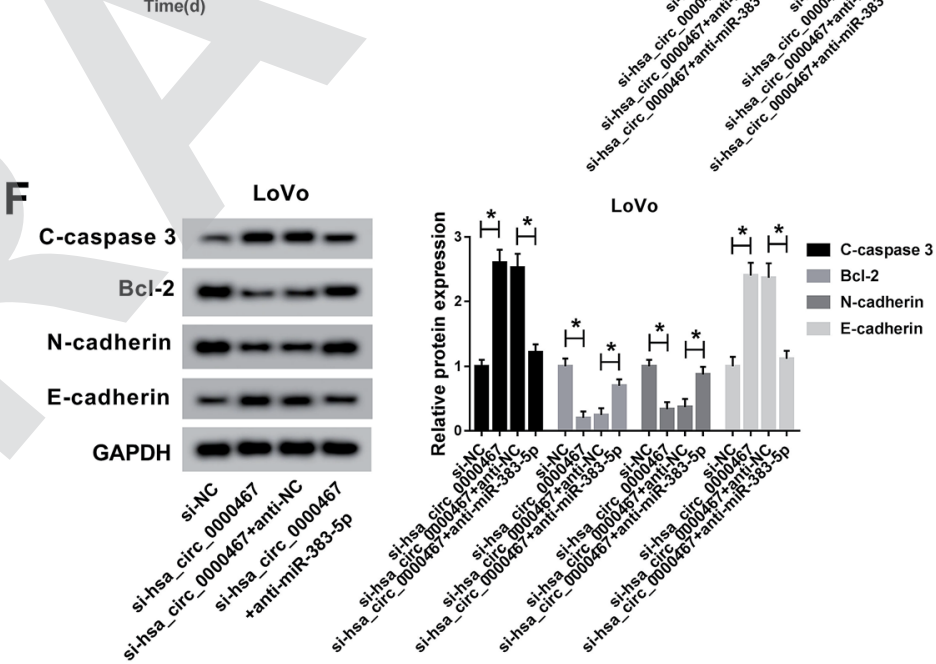

C
E

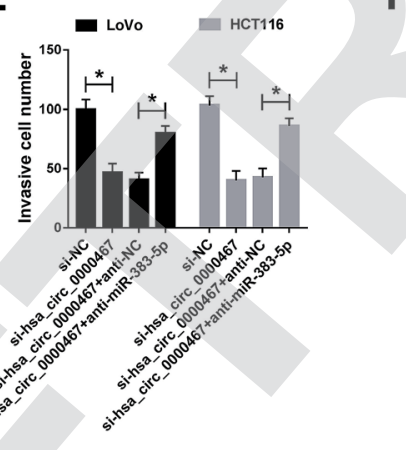

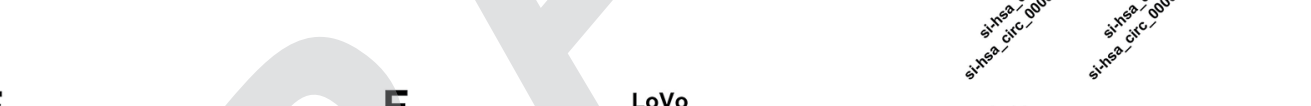
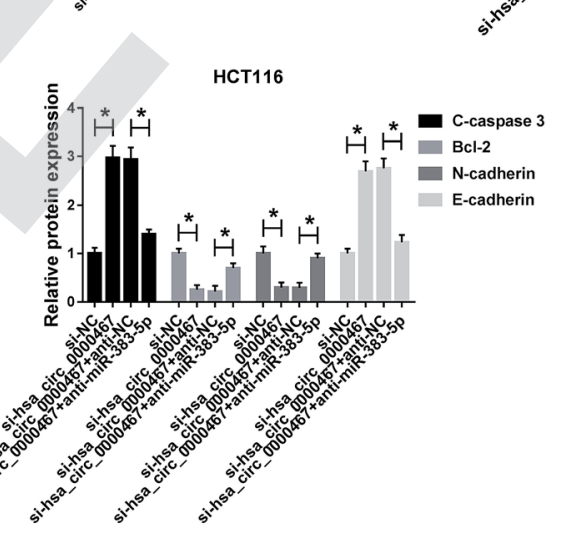

Fig. 4 Hsa_circ_0000467 silencing refrained cell proliferation, metastasis, and EMT but induced apoptosis in LoVo and HCT116 cells by regulating miR-383-5p. (A-G) The LoVo and HCT116 cells were transfected with si-NC, si-hsa_circ_0000467, si-hsa_circ_0000467 + anti-NC, or si-hsa_circ_0000467 + anti-miR-383-5p. (A) The level of miR-383-5p was examined by qRT-PCR. (B) The cell viability was measured via CCK-8 assay. (C) The apoptotic rate was assessed through flow cytometry. ( $D$ and $E$ ) The migrated and invaded abilities were detected via Transwell assay. ( $\mathrm{F}$ and $\mathrm{G}$ ) The protein levels of $\mathrm{C}$-caspase 3, Bcl-2, $\mathrm{N}$-cadherin, and $\mathrm{E}$-cadherin were tested by western blot assay. ${ }^{*} P<0.05$. 
A

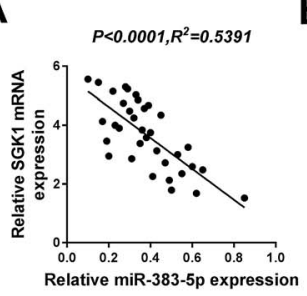

B

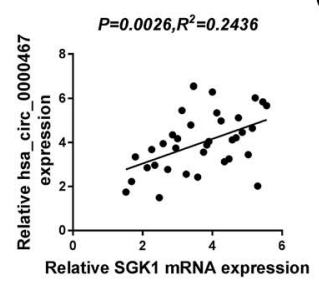

C

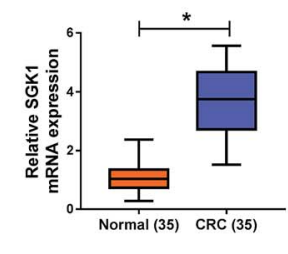

D
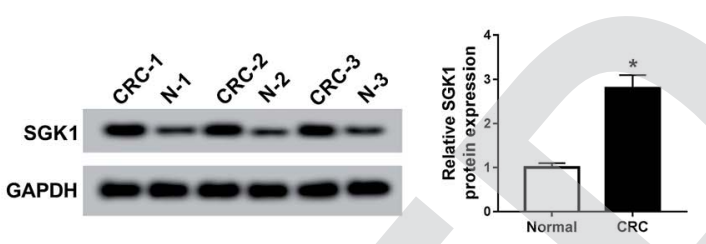

E

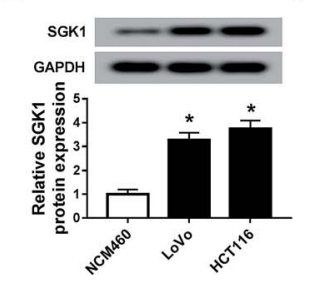

H

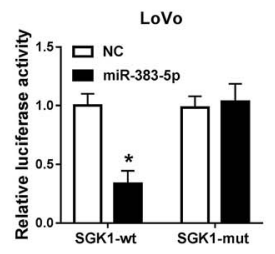

$F$
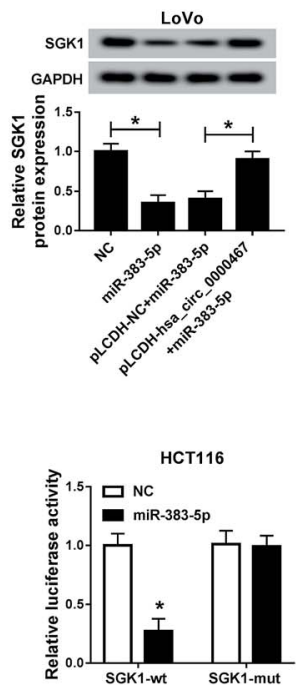

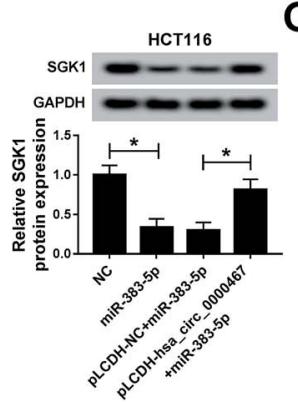

I

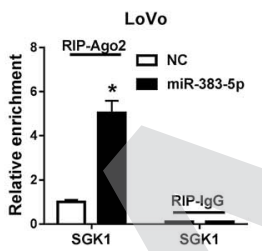

G

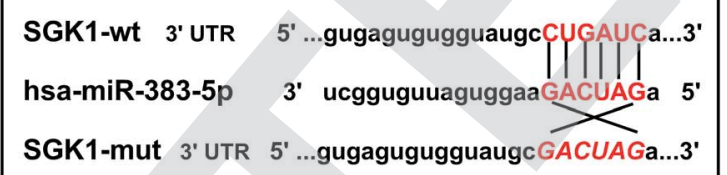

Fig. 5 SGK1 was negatively interacted with miR-383-5p in LoVo and HCT116 cells. (A and B) The correlation between SGK1 and miR-383-5p or hsa_circ_0000467 was processed by Pearson test. (C and D) The MRNA and protein levels of SGK1 in CRC tissues were measured via qRT-PCR and western blot assay, respectively. (E) The protein level of SGK1 in CRC cells was tested by western blot assay. (F) The protein level of SGK1 in LoVo and HCT116 cells transfected with NC, miR-383-5p, pLCDH-NC + miR-383-5p, or pLCDH-hsa_circ_0000467 + miR-383-5p was examined via western blot assay. (G) The complementary binding sites between SGK1 $3^{\prime}$ UTR and SGK1 were presented, as well as the mutant sequences of SGK1 3'UTR. (H) The luciferase activity of SGK1-wt or SGK1-mut reporter in LoVo and HCT116 cells transfected with miR-383-5p or NC was assessed via dual-luciferase reporter assay. (I) The enrichment of SGK1 by Ago2 or IgG in LoVo and HCT116 cells transfected with miR383-5p or NC was tested by RIP assay. ${ }^{*} P<0.05$

dramatically decreased in LoVo and HCT116 cells transfected with miR-383-5p related to that in NC group; however, the luciferase activity of SGK1-mut reporter had no marked fluctuation in any group (Fig. $5 \mathrm{H}$ ). In addition, Ago2 enriched much more miR-383-5p in LoVo and HCT116 cells transfected with SGK1 than that in IgG group (Fig. 5I). Taken together, SGK1 was a candidate target of miR-383-5p in LoVo and HCT116 cells.

MiR-383-5p overexpression impeded cell proliferation, metastasis, and EMT but induced apoptosis in LoVo and HCT116 cells by modulating SGK1

To illustrate whether the effects of miR-383-5p on CRC development were mediated via SGK1, miR-383-5p and SGK1 were transfected into LoVo and HCT116 cells. Firstly, the protein level of SGK1 was obviously reduced in LoVo and HCT116 cells transfected with miR-383-5p, but apparent rescued by the emergence of SGK1 (Fig. 6A). Furthermore, the transfection of miR-383-5p contributed to the striking decrease of cell viability, migrated and invaded abilities in LoVo and HCT116 cells, while the re-introduction of SGK1 relieved these inhibitory impacts (Fig. 6B, D and E). While the facilitated effect on apoptotic rate by miR-383-5p in LoVo and HCT116 cells was mitigated with the introduction of SGK1 (Fig. 6C). In addition, the transfection of SGK1 attenuated the accelerated impact on the protein levels of C-caspase 3, E-cadherin, as well as the restraint effect on the protein levels of Bcl-2, N-cadherin in LoVo and HCT116 cells induced by miR-383-5p (Fig. $6 \mathrm{~F}$ and G). These results indicated that the overexpression of miR-383-5p suppressed cell proliferation, metastasis, and EMT while induced apoptosis in LoVo and HCT116 cells by regulating SGK1.

Hsa_circ_0000467 depletion retarded xenograft tumor growth in vivo

To further validate the effects of hsa_circ_0000467 on CRC progression, HCT116 cells stably transfected with shhsa_circ_0000467 or sh-NC were implanted into nude mice. After $25 \mathrm{~d}$ cultivation, the volume and weight of tumor were notably decreased in sh-hsa_circ_0000467 group compared to that in sh-NC group (Fig. 7A and B). Besides, the level of shhsa_circ_0000467 was remarkably declined in shhsa_circ_0000467 group, while miR-383-5p was conspicuously up-regulated in sh-hsa_circ_0000467 group (Fig. 7C and D). The 
A

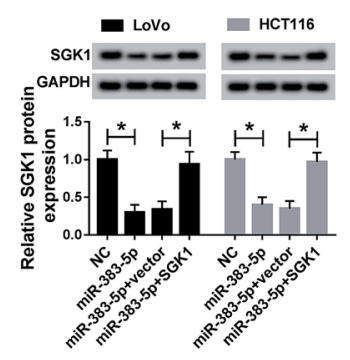

B
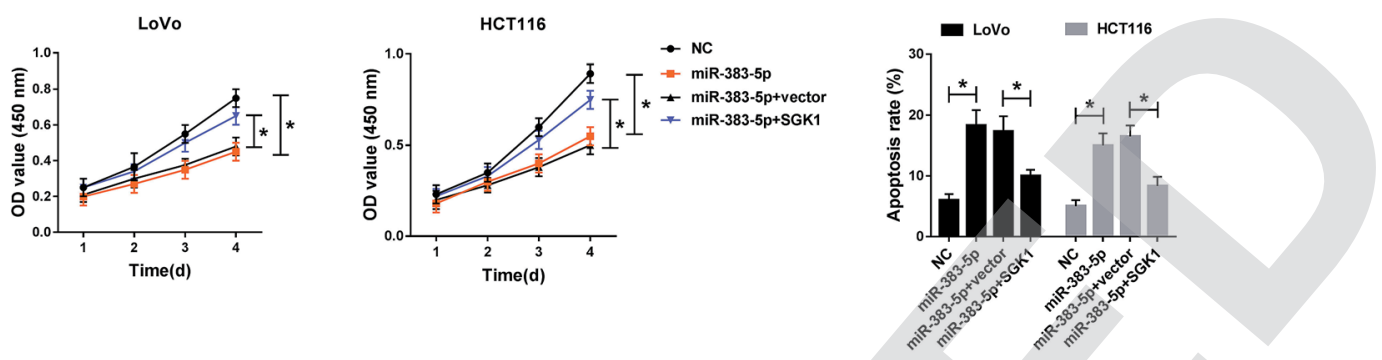

D

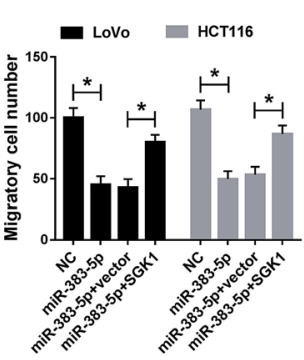

$\mathbf{E}$

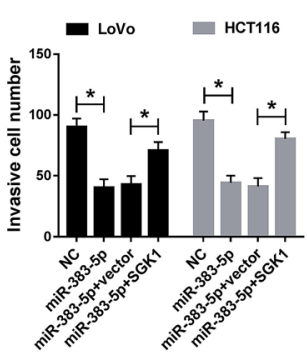

$\mathbf{F}$

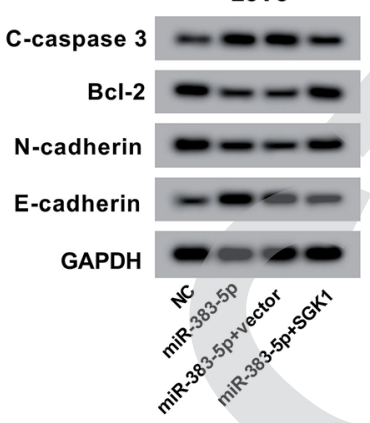

C

G
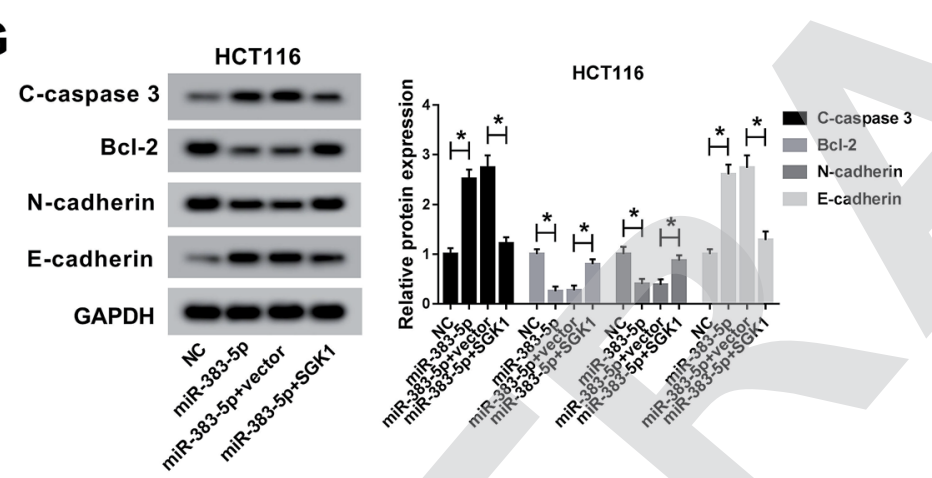

Fig. 6 MiR-383-5p overexpression impeded cell proliferation, metastasis, and EMT but induced apoptosis in LoVo and HCT116 cells by modulating SGK1. (A-G)The LoVo and HCT116 cells were transfected with NC, miR-383-5p, miR-383-5p + vector, or miR-383-5p + SGK1. (A) The protein level of SGK1 was evaluated by western blot assay. (B) The cell viability was tested via CCK- 8 assay. (C) The apoptotic rate was examined through flow cytometry. ( $D$ and $E$ ) The migrated and invaded abilities were measured by Transwell assay. (F and G) The protein levels of C-caspase 3, Bcl-2, N-cadherin, and E-cadherin were detected via western blot assay. $* P<0.05$.

protein level of SGK1 was strikingly reduced in shhsa_circ_0000467 group (Fig. 7E). In addition, the protein levels of C-caspase 3, E-cadherin was evidently elevated, while the protein levels of PCNA, N-cadherin were greatly decreased in sh-hsa_circ_0000467 group (Fig. 7F). The regulatory mechanism of circ_0000467/miR-383-5p/SGK2 in CRC was exhibited in Fig. 8. These data implicated that the silencing of hsa_circ_0000467 blocked xenograft tumor growth in vivo.

\section{Discussion}

Tumor progression is a complicated process in human body. Emerging evidence demonstrated that circRNAs played important roles in tumor progression. ${ }^{21}$ In this research, we aimed to illustrate the effects of hsa_circ_0000467 on CRC progression. These data revealed that hsa_circ_0000467 knockdown repressed cell proliferation, migration, invasion, and EMT while facilitated apoptosis in CRC via miR-383-5p/SGK1 axis.

Accumulating data indicated that the ectopic expression of circRNAs was related to CRC progression. For instance, a study in CRC disclosed that the level of hsa_circ_0000069 was upregulated in CRC, and its knockdown confined cell proliferation, metastasis, and cell cycle progression in vitro. ${ }^{22}$ Another report in CRC demonstrated that hsa_circ_0071589 was highly expressed in CRC; besides, the silencing of hsa_circ_0071589 impeded cell mobility. ${ }^{23}$ Also, Lu et al. reported that hsa_circ_0000467 was up-regulated in gastric cancer, and its depletion curbed cell growth, metastasis while aggravated apoptosis in vitro. ${ }^{10}$ In this exploration, hsa_circ_0000467 was verified as a circRNA and elevated in CRC. The silencing of hsa_circ_0000467 refrained cell growth, metastasis, and EMT while promoted apoptosis in CRC cells. More importantly, 
A

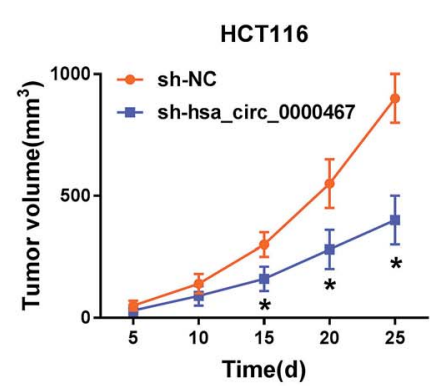

B

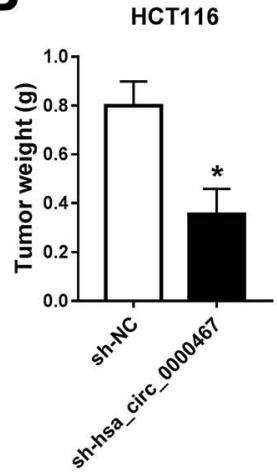

C

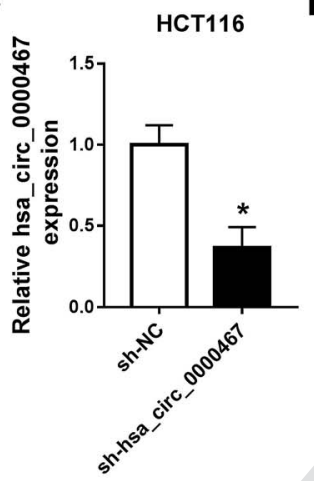

D

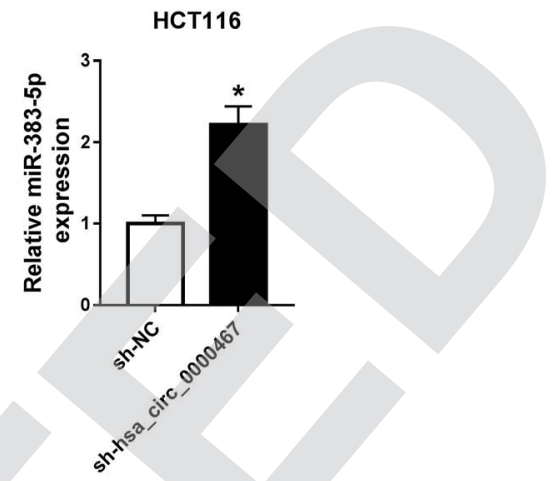

E
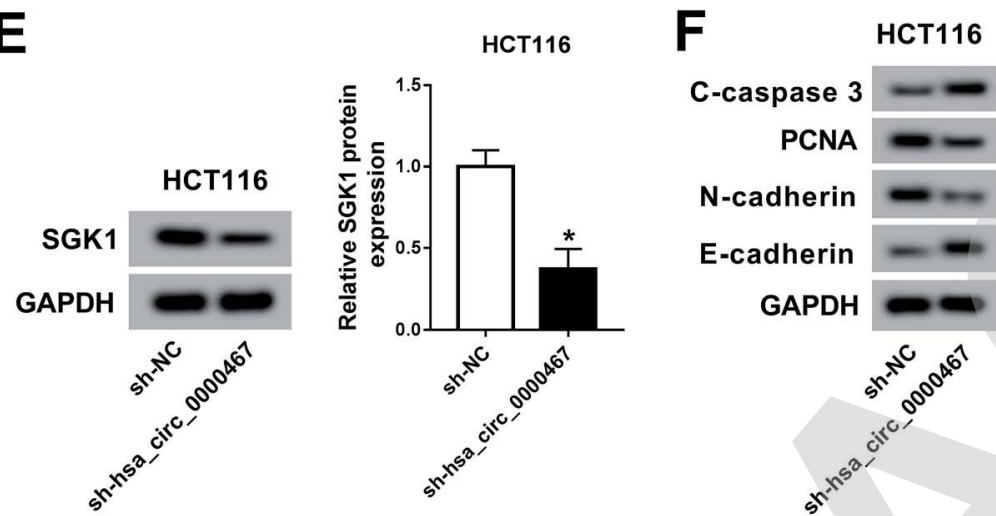

Fig. 7 Hsa_circ_0000467 depletion retarded xenograft tumor growth in vivo. (A-F) The nude mice were injected with HCT116 cells transfected with sh-hsa_circ_0000467 or sh-NC. (A and B) The volume and weight of xenograft tumor were measured. (C and D) The levels of hsa_circ_0000467 and miR-383-5p were examined by qRT-PCR. (E and F) The protein levels of SGK1, C-caspase 3, PCNA, N-cadherin, and Ecadherin were estimated via western blot assay. $* P<0.05$.

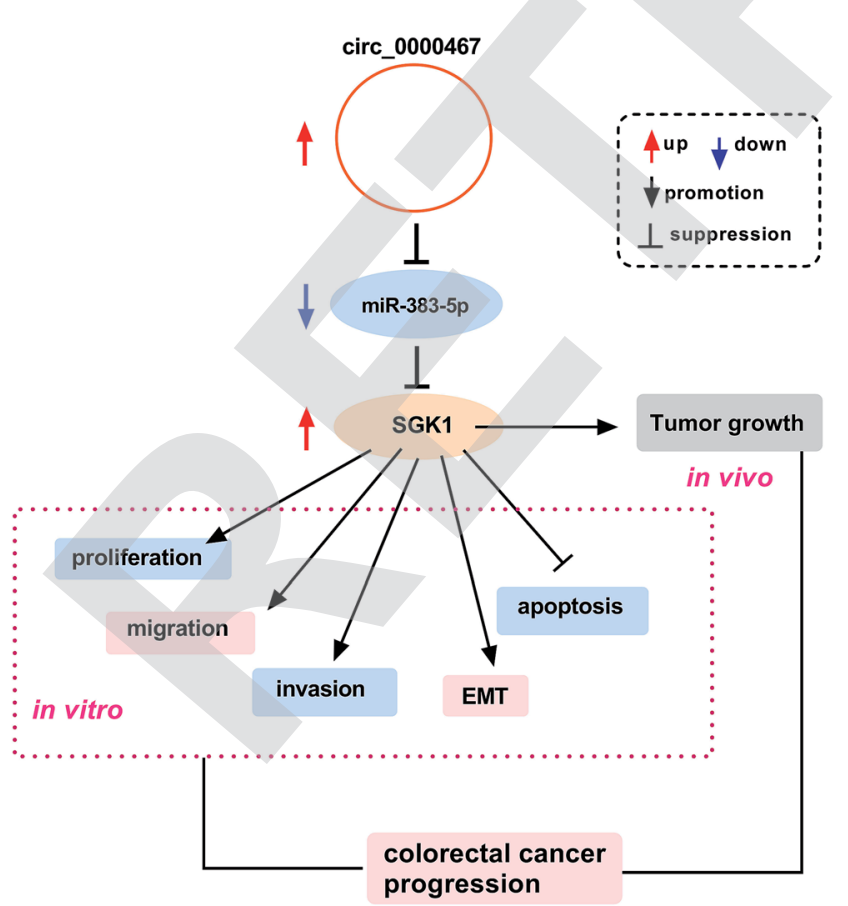

Fig. 8 The flow chart of the mechanism of Hsa_circ_0000467 modulates SGK1 in colorectal cancer cells. hsa_circ_0000467 depletion hampered tumor growth in vivo. These data uncovered that hsa_circ_0000467 knockdown suppressed CRC progression.

Recent studies demonstrated that miR-383-5p was associated with tumor progression. For example, an exploration in hepatocellular carcinoma indicated that miR-383-5p constrained cell growth by regulating AKR1B10 expression. ${ }^{24}$ Another document in lung cancer implied that miR-383-5p regulated lung cancer cell behaviors by targeting CIP2A. ${ }^{25}$ In the current study, miR-383-5p was declined in CRC and validated to be sponged by hsa_circ_0000467. Moreover, hsa_circ_0000467 constrained cell viability, mobility but accelerated apoptosis in CRC cells by modulating miR-383-5p. The above results of miR-383 were in line with previous studies. ${ }^{15,16}$ These results unraveled that hsa_circ_0000467 knockdown refrained CRC progression by sponging miR-383-5p.

Convincing evidence illustrated that the abnormal expression of SGK1 was also implicated in tumor progression. For example, an exploration in prostate cancer revealed that SGK1 knockdown repressed cell growth, metastasis, and EMT in prostate cancer cells. ${ }^{26}$ Another study in lung adenocarcinoma implied that SGK1 facilitated cell migration and invasion mediated by miR-576-3p. ${ }^{27}$ In the present research, SGK1 was highly expressed in CRC and verified as a target of miR-383-5p. Hsa_circ_0000467 silencing inhibited SGK1 expression in CRC 
cells via miR-383-5p. Furthermore, miR-383-5p overexpression confined cell proliferation, metastasis, and EMT but induced apoptosis in CRC cells by regulating SGK1. The results of SGK1 in CRC were consistent with reported studies. ${ }^{18,19}$ These results unraveled that hsa_circ_0000467 depletion retarded SGK1 to impede cell proliferation, migration, invasion, and EMT while promoted apoptosis in CRC cells via miR-383-5p.

\section{Conclusion}

Hsa_circ_0000467, SGK1 were elevated while miR-383-5p was declined in CRC. The functional and mechanistical experiments elucidated that hsa_circ_0000467 modulated SGK1 to accelerate CRC progression by sponging miR-383-5p.

\section{Funding}

This work was supported by Liaoning Natural Science Foundation (20170541015)

\section{Conflicts of interest}

The authors have no conflict of interest to declare.

\section{References}

1 R. L. Siegel, K. D. Miller and A. Jemal, Ca-Cancer J. Clin., 2016, 66, 7-30.

2 S. Manfredi, C. Lepage, C. Hatem, O. Coatmeur, J. Faivre and A.-M. Bouvier, Ann. Surg., 2006, 244, 254.

3 Y. Wang, Y. Mo, Z. Gong, X. Yang, M. Yang, S. Zhang, F. Xiong, B. Xiang, M. Zhou and Q. Liao, Mol. Cancer, 2017, 16, 25.

4 J. N. Vo, M. Cieslik, Y. Zhang, S. Shukla, L. Xiao, Y. Zhang, Y.-M. Wu, S. M. Dhanasekaran, C. G. Engelke and X. Cao, Cell, 2019, 176, 869-881.

5 Y.-H. Luo, X.-Z. Zhu, K.-W. Huang, Q. Zhang, Y.-X. Fan, P.-W. Yan and J. Wen, Biomed. Pharmacother., 2017, 96, 892-898.

6 C. Jin, A. Wang, L. Liu, G. Wang and G. Li, J. Cell. Physiol., 2019, 234, 7247-7256.

7 X. Lu, Y. Yu, F. Liao and S. Tan, Med. Sci. Monit., 2019, 25, 6872-6883.

8 J. Cao, L. Ma, Y. Zhang, H. Guo, X. Niu and T. Zhao, Eur. Rev. Med. Pharmacol. Sci., 2019, 23, 4730-4737.
9 Y. Jin, Y. Ren, Y. Gao, L. Zhang and Z. Ding, Eur. Rev. Med. Pharmacol. Sci., 2019, 23, 3311-3319.

10 J. Lu, P. y. Zhang, J. w. Xie, J. b. Wang, J. x. Lin, Q. y. Chen, L. l. Cao, C. m. Huang, P. Li and C. h. Zheng, J. Clin. Lab. Anal., 2019, 33, e22726.

11 Y. Huang, X. J. Shen, Q. Zou, S. P. Wang, S. M. Tang and G. Z. Zhang, J. Physiol. Biochem., 2011, 67, 129-139.

12 Z. Zhang, J. Li, Y. Huang, W. Peng, W. Qian, J. Gu, Q. Wang, T. Hu, D. Ji and B. Ji, Cell Proliferation, 2018, 51, e12505.

13 L. Wang, F. Jiang, F. Ma and B. Zhang, J. Cell Commun. Signal., 2019, 1-12.

14 Y. Zhang, M. Sun, Y. Chen and B. Li, Front. Pharmacol., 2019, 10, 741.

15 J. Li, A. R. Smith, R. T. Marquez, J. Li, K. Li, L. Lan, X. Wu, L. Zhao, F. Ren and Y. Wang, Mol. Carcinog., 2018, 57, 1408-1420.

16 F. Yan, Z. Tu, L. Duan, D. Wang and F. Lin, Mol. Med. Rep., 2018, 17, 6893-6901.

17 M. K. Webster, L. Goya, Y. Ge, A. Maiyar and G. Firestone, Mol. Cell. Biol., 1993, 13, 2031-2040.

18 X. Liang, C. Lan, G. Jiao, W. Fu, X. Long, Y. An, K. Wang, J. Zhou, T. Chen and Y. Li, Exp. Mol. Med., 2017, 49, e399.

19 T. Liu, T. Yu, H. Hu and K. He, Biomed. Pharmacother., 2018, 98, 286-296.

20 J. Tangir, N. Bonafé, M. Gilmore-Hebert, O. Henegariu and S. K. Chambers, Clin. Exp. Metastasis, 2004, 21, 477-483.

21 R. Zhou, Y. Wu, W. Wang, W. Su, Y. Liu, Y. Wang, C. Fan, X. Li, G. Li and Y. Li, Cancer Lett., 2018, 425, 134-142.

22 J.-n. Guo, J. Li, C.-l. Zhu, W.-t. Feng, J.-x. Shao, L. Wan, M.-d. Huang and J.-d. He, OncoTargets Ther., 2016, 9, 7451.

23 W. Yong, X. Zhuoqi, W. Baocheng, Z. Dongsheng, Z. Chuan and S. Yueming, Biomed. Pharmacother., 2018, 102, 11881194.

24 J. Wang, Y. Zhou, X. Fei, X. Chen and Y. Chen, Sci. Rep., 2018, 8, 11094.

25 S. Zhao, X. Gao, S. Zang, Y. Li, X. Feng and X. Yuan, Oncol. Lett., 2017, 14, 3573-3579.

26 W. Liu, X. Wang, Y. Wang, Y. Dai, Y. Xie, Y. Ping, B. Yin, P. Yu, Z. Liu and X. Duan, J. Exp. Clin. Cancer Res., 2018, 37, 73.

27 E. J. Greenawalt, M. D. Edmonds, N. Jain, C. M. Adams, R. Mitra and C. M. Eischen, Mol. Cancer Res., 2019, 17, 289-298. 